\title{
Connaître les cyclooxygénases pour mieux contrôler les effets des anti-inflammatoires non stéroïdiens
}

L'aspirine est un médicament étonnamment puissant, au champ d'action très étendu puisqu'il est utilisé pour soulager les douleurs, la fièvre et les phénomènes inflammatoires, prévenir les thromboses coronaires et cérébrales, avec des effets secondaires tels que des troubles ulcéreux de l'estomac, des rétentions liquidiennes par les reins et un effet anticoagulant. En biologie cellulaire, on a montré qu'il inhibe les transports ioniques à travers les membranes biologiques et interfère avec l'activation des globules blancs; au niveau moléculaire, il active les gènes des heat shock proteins chez la drosophile !

On a attendu longtemps l'élucidation de ses mécanismes d'action. C'est dans les années 1970 que John Vane (qui a obtenu le prix Nobel en 1982) a proposé le premier mécanisme d'action satisfaisant: l'aspirine inhibe l'enzyme permettant la synthèse des prostaglandines [1], hormones locales dérivées de l'acide arachidonique, acide gras commun des membranes cellulaires. Par la suite, il a été montré que tous les médicaments doués des propriétés de l'aspirine, les antiinflammatoires non stéroïdiens (AINS), bloquent la synthèse des prostaglandines. Les prostaglandines isolées et injectées provoquent les signes cardinaux de l'inflammation : fièvre, douleur, chaleur. Dans l'estomac, elles règlent l'acidité locale et la synthèse du mucus; dans les plaquettes, elles sont représentées par le thromboxane $B_{2}$, puissant agent agrégant et vasoconstricteur; dans les cellules endothéliales, leur dérivé, la prostacycline, est un vasodilatateur.

L'enzyme cible des AINS est la prostaglandine synthase ou cyclooxygénase (COX) qui convertit l'acide arachidonique en prostaglandine $\mathrm{H}_{2} \quad\left(\mathrm{PGH}_{2}\right) ;$ du métabolisme de
$\mathrm{PGH}_{2}$ dérivent ensuite les diverses prostaglandines, prostacyclines et thromboxanes. Il existe au moins deux isoformes de $\mathrm{COX}$, de même poids moléculaire et avec environ $60 \%$ de similitude. COX-1 est physiologique, exprimée de façon constitutive. Son activation conduit à la production de prostacycline qui, libérée par l'endothélium est antithrombogénique [2], alors que, libérée par la muqueuse gastrique, elle protège les cellules d'un excès d'acidité et active la synthèse de mucus [3]. COX-2, au contraire, est inductible par les agents proinflammatoires (cytokines, mitogènes, endotoxines) [4] (Tableau I).

L'aspirine, l'indométhacine, le piroxicam sont 20 à 50 fois plus actifs sur COX-1 que sur COX-2, alors que le diclofénac, le naproxène, le méclofénamate, l'ibuprofène ont une activité voisine sur les deux isoformes $[5,6]$ (figure 1). Ces dernières années, un important effort de recherche pharmacologique a été réalisé pour mettre au point des AINS inhibiteurs sélectifs de COX-2. C'est la cible thérapeutique la plus intéressante à développer actuellement: l'aspirine et les autres AINS sur le marché n'ont qu'une faible affinité pour COX-2, expliquant la nécessité de fortes doses de médicament pour obtenir l'action anti-inflammatoire, alors que leurs effets secondaires indésirables (irritation de l'estomac, effets toxiques sur le rein) sont tous liés à leur inhibition de COX-1 sur laquelle ils sont très actifs.

Un pas en avant dans la compréhension du mode d'action de COX a été franchi lors de la résolution tridimensionnelle de COX-1 [7]. La protéine a trois domaines distincts, indépendants dans leur repliement: un petit domaine compact, dont la conformation et l'arrangement des ponts disulfures le rendent très simi- laire à l'EGF (epidermal growth factor); un motif de liaison à la membrane : l'enzyme s'insère uniquement dans le feuillet interne de la bicouche lipidique des membranes (essentiellement le réticulum endoplasmique) ; et un domaine enzymatique, avec deux sites catalytiques adjacents et distincts, pour l'activité cyclooxygénase (qui transforme l'acide arachidonique en $\mathrm{PGG}_{2}$ ) et l'activité peroxydase (qui transforme $\mathrm{PGG}_{2}$ en $\mathrm{PGH}_{2}$ ). Trois hélices bornent l'entrée d'un canal étroit, domaine catalytique de l'activité cyclooxygénase, permettant à l'acide arachidonique d'accéder au site actif à partir de l'intérieur de la bicouche. Les sites d'action des AINS actuels ont été en partie dévoilés. Il est vraisemblable que la structure de COX-2 sera bientôt connue et que la recherche d'un inhibiteur spécifique de COX-2 sera facilitée.

Quoi qu'il en soit, l'aspirine est de plus en plus utilisée pour son action anticoagulante : à faible dose, l'aspirine n'agit que sur les plaquettes. Elle inactive COX-1 de manière irréversible et la plaquette ne peut ni la régénérer ni la resynthétiser pour la fin de sa durée de vie. En revanche, la cellule endothéliale peut synthétiser les nouvelles molécules de COX-1 nécessaires à la production de prostacycline. Ajoutons que l'aspirine est absorbée dans la circulation présystémique où elle agit sur les plaquettes avant de se retrouver en beaucoup plus faible concentration dans le flux artériel où elle épargnera la cellule endothéliale. En réduisant la formation de thromboxane $\mathrm{A}_{2}$ par les plaquettes, elle permet une réduction importante de l'agrégation plaquettaire, base de son utilisation dans la prévention des accidents vasculaires. De nombreux essais thérapeutiques ont montré son efficacité pour prévenir l'infarctus du myocarde (ISIS 2 [8], 


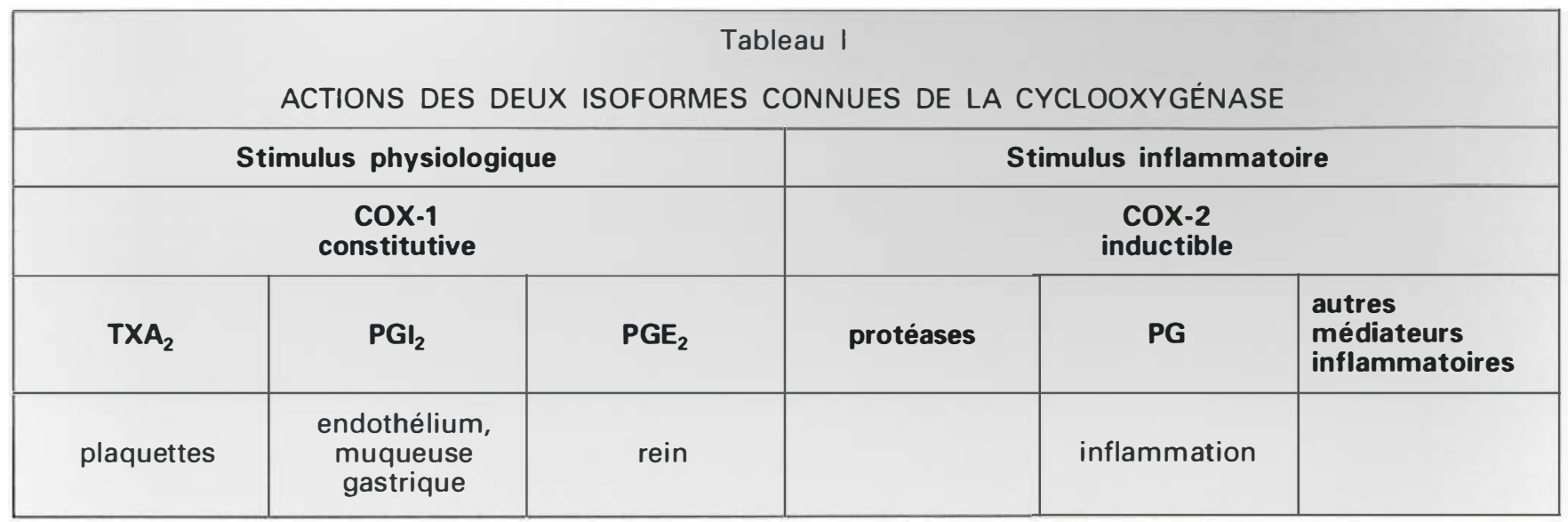

La cyclooxygénase transforme l'acide arachidonique en prostaglandine $\mathrm{H}_{2}$, métabolisée ensuite en divers eicosanoïdes ldérivés d'acides gras à 24 carbones). Dans les conditions physiologiques, l'activation de COX-1 dans les plaquettes, l'endothélium ou le rein entraîne la libération de thromboxane $A_{2}\left(T X A_{2}\right)$, prostacycline $\left(P G I_{2}\right)$, ou prostaglandine $E_{2}\left(P G E_{2}\right)$. La libération de ces eicosanoïdes est spécifiquement inhibée par l'aspirine et autres anti-inflammatoires non stéroïdiens (AINS). A la suite d'un stimulus inflammatoire, les cytokines libérées (IL1 par exemple) induisent la synthèse de COX-2 dans les macrophages ou autres cellules participant au processus inflammatoire et la libération de prostaglandines (PG), en même temps que des protéases et d'autres médiateurs de l'inflammation (radicaux oxygénés par exemple). La voie de la COX-2 peut être interrompue à divers niveaux par les antagonistes ou anticorps des cytokines et des mitogènes, par les inhibiteurs de son induction (glucocorticoïdes) ou par des inhibiteurs spécifiques de COX-2, comme l'aspirine à forte dose. Les effets anti-inflammatoires de l'aspirine sont dus à l'inhibition de COX-2, tandis que ses effets anti-agrégants plaquettaires mais aussi ses effets secondaires non désirables proviennent de l'inhibition de COX-1.

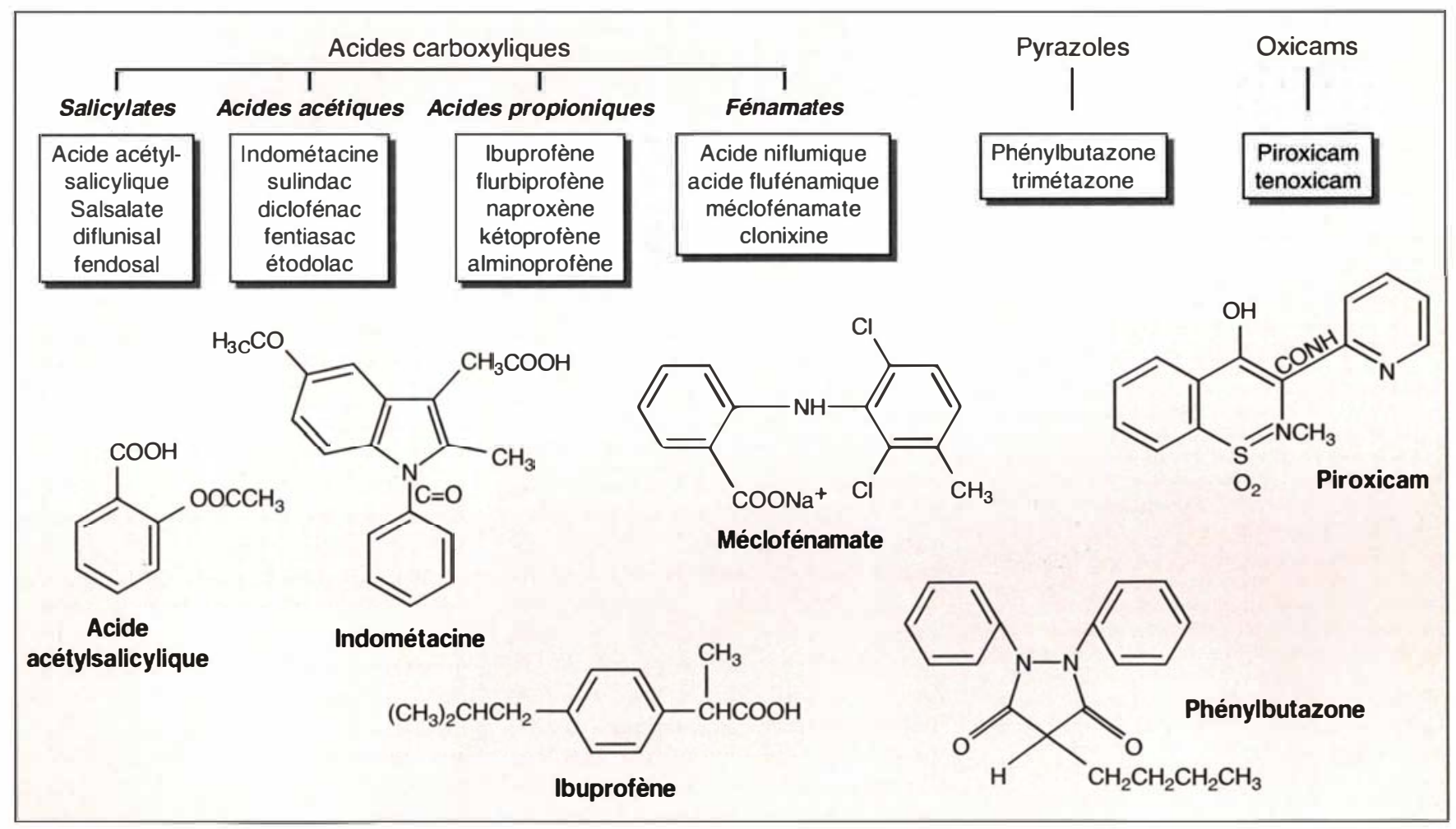

Figure 1. Les anti-inflammatoires non stéroïdiens. 
ISIS $3\left(\mathrm{~m} / \mathrm{s} n^{\circ} 7\right.$, vol. $8, p$. 735)) et les thromboses cérébrales [9], pour éviter les resténoses après angioplastie [10], en traitement de la toxémie gravidique [11, 12]. Ajoutons que le faible coût de ce médicament, à l'époque actuelle de dérapage des coûts de santé, lui confère un attrait non négligeable.

E.B.

1. Vane JR. Inhibition of prostaglandin synthesis as a mechanism of action of aspirin-like drugs. Nature 1971 ; 231 : 232-5.

2. Moncada S, Gryglewski R, Bunting S, Vane $J R$. An enzyme isolated from arteries transforms prostaglandin endoperoxides to an unstable substance that inhibits platelet aggregation. Nature 1976; 263 : 663-5.

3. Whittle BJR, Higgs GA, Eakins KE, Moncada $S$, Vane JR. Selective inhibition of prostaglandin production in inflammatory exudates and gastric mucosa. Nature 1980 ; 284 : 271-3.

4. Lee SH, Soyoola E, Chanmugam P, Hart S, Sun W, Zhong H, Liou S, Simmons D, Hwang D. Selective expression of mitogen-inducible cyclooxygenase in macrophages stimulated with lipopolysaccharide. J Biol Chem 1992; 267 : 25934-8.

5. Meade EA, Smith WL, DeWitt DL. Differential inhibition of prostaglandin endoperoxide synthase (cyclooxygenase) isozymes by aspirin and other non-steroidal anti-inflammatory drugs. J Biol Chem 1993; 268: 6610-4.

6. Mitchell JA, Akarasereenont P, Thiemermann C, Flower RJ, Vane JR. Selectivity of nonsteroidal antiinflammatory drugs as inhibitors of constitutive and inducible cyclooxygenase. Proc Natl Acad Sci USA 1993 ; 90 : 11693-7.

7. Picot D, Loll PJ, Garavito RM. The X-ray crystal structure of the membrane protein prostaglandin $\mathrm{H}_{2}$ synthase-1. Nature 1994; 367 : 243-9.

8. ISIS-2 (second international study of infarct survival) collaborative group. Randomised trial of intravenous streptokinase, oral aspirin, both, or neither among 17187 cases of suspected acute myocardial infarction: ISIS-2. Lancet $1988 ; 2: 349-60$.

9. Ranke C, Hecker H, Creutzig A, Alexander $\mathrm{K}$. Dose-dependent effect of aspirin on carotid atherosclerosis. Circulation 1993 ; 87 : 1873-9. 10. Van der Meer, J, Hillege HL, Koostra GJ, Ascoop CA, Pfisterer M, van Gilst WH, Lie KI. Prevention of one-year vein-graft acclusion after aortocoronary-bypass surgery : a comparison of low-dose aspirin, low-dose aspirin plus dipyridamole, and oral anticoagulants. The CABADAS research group of the interuniversity cardiology institute of the Netherlands. Lancet 1993 ; 342 : 257-64.

11. Beaufils M, Densimons R, Uzan S, Colon JC. Prevention of preeclampsia by early antiplatelet therapy. Lancet $1985 ; 1: 840-2$.

12. Uzan S, Beaufils M, Breart G, Bazin B, Capitant C, Paris J. Prevention of fetal growth retardation with low-dose aspirin : findings of

口n BRÈVES

Disque accru de tétanos néonatal en zone d'endémie palustre. Le tétanos néonatal est fréquent dans les régions d'endémie palustre. Cependant, les relations entre les deux maladies sont mal explicitées. Un travail récent a été effectué dans un hôpital de campagne en Nouvelle-Guinée par l'équipe de l'École de médecine tropicale de Liverpool [1]. Un suivi des femmes enceintes y est réalisé de façon très régulière, et comporte systématiquement une vaccination contre le tétanos et une chimioprophylaxie antipaludéenne par la chloroquine. L'étude a porté sur 224 femmes suivies jusqu'à l'accouchement, divisées en trois groupes selon le taux de parasitémie maternelle, reflet de l'infestation placentaire. Aucune différence significative n'a été retrouvée en ce qui concerne le poids des enfants ou la durée de gestation. Cependant, si l'on compare les taux d'anticorps antitétaniques trouvés chez les nouveau-nés, les différences sont extrêmement importantes. Pour tenir compte des différences interindividuelles, ce taux d'immunité passive transmise a été dans chaque cas rapporté à celui qu'on observait chez la mère (pour $1 \mathrm{UI} / \mathrm{ml})$. Les résultats sont les suivants. En cas de placenta très infesté ( $>35$ parasites pour 200 leucocytes), à $1 \mathrm{UI} / \mathrm{ml}$ chez la mère correspond une moyenne de $0,18 \mathrm{UI} / \mathrm{ml}$ chez l'enfant. Si le placenta est moins infesté $(<35$ parasites pour 200 leucocytes), l'immunité passive est un peu plus élevée chez l'enfant, $0,23 \mathrm{UI} / \mathrm{ml}$ pour $1 \mathrm{UI} / \mathrm{ml}$ chez la mère. Dans le groupe non parasité, on retrouve chez l'enfant un taux nettement plus élevé, 0,82 UI pour $1 \mathrm{UI} / \mathrm{ml}$ chez la mère, c'està-dire un passage adéquat transplacentaire de l'immunité passive. Le mécanisme expliquant ces données n'est pas évident. L'infestation placentaire pourrait induire un retard de croissance fotale et influer sur le passage des anticorps maternels à l'enfant. On peut aussi évoquer l'augmentation non spécifique des IgG chez les mères impaludées, et la limite au transport que représenterait le nombre limité de récepteurs placentaires.

[1. Brair ME, et al. Lancet 1994 ; 343 : 208-9.]

Maladie d'Alzheimer et les isoformes de l'apoliprotéine $E$. Comme nous l'avons rapporté dans médecine/sciences une liaison génétique a été mise en évidence entre la présence de l'allèle 4 de l'ApoE et la susceptibilité à l'Alzheimer dans les cas sporadiques comme dans les formes familiales $\left(\mathrm{m} / \mathrm{s} n^{\circ} 10\right.$, vol. $\left.9, p .1142\right)$. Un cas intéressant d'Alzheimer familial vient d'être rapporté [1]. Dans une grande famille a été mise en évidence une mutation du gène codant pour le précurseur de la protéine $\beta$ amyloïde (chromosome 21); tous les membres de la famille ayant hérité l'allèle muté sont atteints par la maladie à un âge très régulier (47 \pm 3 ans). Tous, sauf un qui n'a encore aucun signe de la maladie à un âge supérieur de plus de deux écarts-types. L'étude du génotype de l'apolipoprotéine $\mathrm{E}$ a montré la présence de l'allèle 4 chez tous les porteurs de la mutation sauf chez le sujet cliniquement indemne. Cette observation conforte l'hypothèse d'une interaction entre les produits des deux gènes, ApoE-4 et protéine $\beta$ amyloïde mutée. En revanche, aucune relation n'a été montrée entre le génotype $A p o E$ et l'âge du début de la maladie dans une grande famille ayant un locus pathogène sur le chromosome 14 dont le gène n'est pas encore identifié. [1. Saint George-Hyslop P, et al. Science 1994; 263: 537.] 